\title{
Prevalencia de Maloclusiones dentales en escolares de 12 años en la parroquia Bellavista, Cuenca, 2016
}

\section{Prevalence of dental Maloclusions in schoolchildren of 12 years in the Bellavista parish, Cuenca, 2016}

\author{
Astudillo Correa, Sonya del Cisne* y González Campoverde, Lorena Alexandra \\ Universidad Católica de Cuenca, Ecuador \\ *cisnecita@outlook.com
}

\begin{abstract}
Resumen
El objetivo del presente estudio fue determinar la prevalencia de maloclusiones en escolares de 12 años de la parroquia urbana Bellavista de la ciudad de Cuenca-Ecuador. Es un estudio observacional, transversal y exploratorio en el cual se observaron 111 escolares de 12 años de los cuales 62 fueron mujeres (56\%) y 49 varones (44\%), el estudio se realizó previo a la calibración de sus examinadores, consentimiento de sus padres y el asentimiento de los escolares. Todos los exámenes se realizaron con luz natural, antes del recreo y cuidando los aspectos de asepsia y antisepsia. Todos los datos se registraron en la ficha epidemiológica de la Universidad Católica de Cuenca. La prevalencia de maloclusiones fue del $77 \%$ en el total de escolares; el $23 \%$ de escolares registró una normooclusión; al relacionarla según el sexo el género femenino demostró una mayor prevalencia con el $44 \%$ que el sexo masculino con el $33 \%$. En este estudio se concluyó que existe una significativa prevalencia de maloclusiones en los escolares de la parroquia Bellavista de la ciudad de Cuenca, además es más frecuente la presencia de maloclusiones en mujeres que en varones. Respecto a la relación molar la clase I según la clasificación de Angle es la de mayor prevalencia.
\end{abstract}

Palabras clave: Maloclusión, Clase, Prevalencia, Escolares .

\begin{abstract}
The objective of the present study was to determine the prevalence of malocclusions in 12-year-old schoolchildren from the Bellavista urban parish of Cuenca city in Ecuador. It is an bservational, transverse and exploratory study in which were observed 111 schoolchildren of 12 years age, which of them 62 were women (56\%) and 49 boys (44\%), the study was realized before the calibration of their examiners, assent of his parents and the assent of the students. All examinations were realized by natural light, before recess and taking care of the aspects of asepsis and antisepsis. All the information registered in the epidemiological card of the Catholic University of Cuenca. The prevalence of malocclusions was $77 \%$ in the total number of students; $23 \%$ of schoolchildren registered a normal occlusion; When related by sex, the female gender obtained a prevalence of $44 \%$ and the male sex $33 \%$. In this study it was concluded that there is a significant prevalence of malocclusions in the Bellavista parish of Cuenca city, and malocclusion is more frequent in women than in men. Respect of the molar relationship the class I according to Angle's classification is the one with the highest prevalence.
\end{abstract}

Key words: Malocclusion, Class, Prevalence, Schoolchildren .

\section{Introducción}

La Ortodoncia, es la ciencia que se dedica a la prevención, tratamiento y corrección de las anomalías dentomaxilofaciales, esta se encuentra íntimamente relacionada con la oclusión, la cual es la relación eficaz entre los elementos del sistema masticatorio en los que se encuentran: dientes, tejidos de soporte, sistema neuromuscular, articulación temporomandibular y esqueleto cráneofacial. ${ }^{?, 1}$ Los tejidos del sistema masticatorio trabajan de una forma dinámica y conjunta que puede generar cambios funcionales y remodelación de las estructuras anatómicas.
Sin embargo, ante la presencia de contactos prematuros se pueden producir parafunciones y disfunciones en el aparato masticatorio. ${ }^{1,2}$

Las maloclusiones se originan por la existencia de una diferencia entre el tamaño de los dientes y el espacio que hace falta para que se encuentren alineados, produciendo en algunas ocasiones que los dientes no erupcionen y lleguen a quedar impactados internamente en el hueso, o a su vez erupcionen en posición alteradas generando apiñamientos dentarios. ${ }^{1,5}$

Existen múltiples factores para que las maloclusiones se desarrollen, mismos que se clasifican en locales, como: legado, medio ambiente, daños hereditarios y las costum- 
bres nocivas que acogen los niños como morderse las uñas, succionarse los dedos, el uso excesivo del chupón e interponer la lengua. Entre los factores generales, se encuentran alteraciones de número de dientes, tamaño, forma del frenillo lingual, pérdida precoz, erupción tardía de los dientes y restauraciones defectuosas. ${ }^{2,4}$

Se pueden utilizar varios métodos para clasificar las maloclusiones, sin embargo, el método más utilizado es la clasificación de Angle, mismo que se basa en la relación que existe entre la arcada dental inferior con respecto a la superior, tomando como referencia al primer molar permanente. , $^{3,6}$

Las clases esqueléticas I, II y III manifiestan características peculiares consecuencia de la expresión genética a través del crecimiento. Estas características advierten la presencia de alteraciones funcionales ligadas a la bioestructura, entre las cuales se encuentran la deglución, masticación, respiración y el habla. ${ }^{2}$

Las personas con maloclusión clase I poseen un biotipo mesofacial, con una relación maxilomandibular normal, musculatura y perfil blando armónico y un equilibrio entre los ejes verticales y transversales. ${ }^{2,7-11}$

Según Moyers y Uribe, ${ }^{2}$ los pacientes con clase II división 1 presentan una cara alargada con un modelo facial leptoprosopo y con aumento o disminución del tercio facial inferior indicando una mordida abierta o profunda correspondientemente, en cambio, los pacientes clase II división 2, presentan una cara corta con un modelo facial euriprosopo y con disminución de la altura facial inferior, presentando una mordida abierta. ${ }^{2}$

Los pacientes con Clase III presentan: un ángulo de perfil cóncavo superior a $175^{\circ}$, un reborde orbitario hipoplásico, los pómulos se encuentran aplanados o con curvatura invertida, existe una falta de control entre el surco nasal y submentoniana, un aplanamiento del surco mandibular y el labio inferior se encuentra más prominente que el superior. ${ }^{2}$

Según la Organización Mundial de la Salud (OMS) las maloclusiones ocupan el tercer lugar dentro de los problemas de salud oral, considerándose una prevalencia a nivel mundial del $65 \%$ al $89 \%$ de la población, ${ }^{3}$ por ello, el propósito de este trabajo es determinar la prevalencia de maloclusiones en escolares de 12 años en la parroquia Bellavista de la ciudad de Cuenca.

\section{Materiales y Métodos}

El presente es un estudio observacional, transversal y descriptivo. La población fue de 111 escolares de 12 años de edad. ${ }^{12}$ Para realizar el estudio se solicitó el permiso primero de las diferentes instituciones educativas de la parroquia mediante la autorización emitida por el distrito zonal de educación, luego se pidió la autorización de los padres mediante un consentimiento informado y a su vez, se solicitó el asentimiento informado de los escolares para poder iniciar el examen bucal.

Las condiciones para el examen fueron el operador sentado junto al paciente. Se realizó el examen clínico con luz natural y antes de la hora del refrigerio. El instrumental utilizado fue un espejo bucal, una sonda periodontal Carolina del Norte y reglas milimetradas. Se cuidaron todos los aspectos relacionados a la asepsia y la antisepsia, y para cubrir con este fin se utilizaron barreras de protección para el operador. En la realización de todos los exámenes hubo un anotador quien iba corroborando los datos con el examinador.

El examinador acató las recomendaciones de no tocar con los dedos la boca del paciente, en la inspección intraoral siempre utilizó los espejos bucales, el tipo de oclusión se estableció basándose en la clasificación de Angle, misma que toma como referencia las clases molar y canina, además se observó las características propias que presentaba cada escolar para con ello poder clasificarlos en pacientes con: Normooclusión, Clase I, Clase II, Clase II-1, Clase II-2 y Clase III3, ${ }^{12-17}$

Se realizó además un examen minucioso del estado de salud de los tejidos duros y blandos y la existencia de discrepancias dentales dentro de los planos sagital, vertical y transversal, analizando la presencia de: mordida abierta, mordida cruzada anterior y posterior, overjet, overbite y desviación de la línea media. ${ }^{18-22}$

Para analizar el tipo de perfil que posee cada escolar se utilizaron los puntos glabela, subnasal y pogonión blando para determinar el perfil facial anteroposterior; el perfil vertical se obtuvo analizando los planos de Camper y el plano mandibular obteniendo con ello los perfiles normodivergente, hiperdivergente e hipodivergente. ${ }^{2}$ La observación visual clínica del rostro del paciente fue útil para poder realizar un diagnóstico presuntivo de la clase esqueletal del escolar, para obtenerlo se utilizaron las características de la oclusión en su máxima intercuspidación. , $^{2,10,18}$

\section{Resultados}

Con la muestra antes citada se registró que 62 (56\%) corresponden al sexo femenino y $49(44 \%)$ al sexo masculino.

Tabla 1. Maloclusión en escolares de 12 años.

\begin{tabular}{lcccccc}
\hline & \multicolumn{2}{c}{ Femenino } & \multicolumn{2}{c}{ Masculino } & \multicolumn{2}{c}{ Total } \\
& n & \% & n & \% & n & \% \\
\hline Maloclusión & 49 & 44 & 37 & 33 & 86 & 77 \\
Normooclusión & 13 & 12 & 12 & 11 & 25 & 23 \\
Total & 62 & 56 & 49 & 44 & 111 & 110 \\
\hline
\end{tabular}

El $77 \%$ presentó maloclusión siendo más frecuente en el sexo femenino que presentó un $44 \%$ en relación al sexo masculino que registró un $33 \%$, la determinación se la realizó valorando las alteraciones en los tres planos del espacio: sagital, vertical y transversal. (Tabla 1)

La relación molar derecha prevalece la clase I con el $41 \%$ en el sexo femenino y con el $29 \%$ en el sexo masculino; y la relación molar izquierda demostró mayor prevalencia en la clase I del sexo femenino con un $37 \%$ y en el sexo masculino un $32 \%$; encontrándose en menor 
Tabla 2. Relación molar de Angle según el sexo.

\begin{tabular}{|c|c|c|c|c|c|c|c|c|c|c|c|c|c|c|c|c|}
\hline & \multicolumn{8}{|c|}{ Relación Molar Dereccha } & \multicolumn{8}{|c|}{ Relación Molar Izquierda } \\
\hline & \multicolumn{2}{|c|}{ Clase I } & \multicolumn{2}{|c|}{ Clase II } & \multicolumn{2}{|c|}{ Clase III } & \multicolumn{2}{|c|}{ N.A } & \multicolumn{2}{|c|}{ Clase I } & \multicolumn{2}{|c|}{ Clase II } & \multicolumn{2}{|c|}{ Clase III } & \multicolumn{2}{|c|}{ N.A } \\
\hline & $\mathrm{n}$ & $\%$ & $\mathrm{n}$ & $\%$ & $\mathrm{n}$ & $\%$ & $\mathrm{n}$ & $\%$ & $\mathrm{n}$ & $\%$ & $\mathrm{n}$ & $\%$ & $\mathrm{n}$ & $\%$ & $\mathrm{n}$ & $\%$ \\
\hline Femenino & 46 & 41 & 4 & 4 & 8 & 7 & 4 & 4 & 41 & 37 & 8 & 7 & 10 & 9 & 3 & 3 \\
\hline Masculino & 32 & 29 & 6 & 5 & 5 & 5 & 6 & 5 & 35 & 32 & 5 & 5 & 6 & 5 & 3 & 3 \\
\hline Total & 78 & 70 & 10 & 9 & 13 & 12 & 10 & 9 & 86 & 69 & 13 & 12 & 16 & 14 & 6 & 6 \\
\hline
\end{tabular}

porcentaje los que no presentaban relación molar tanto en el sexo femenino como masculino. (Tabla 2)

Tabla 3. Prevalencia de la Clase Esqueletal mediante un diagnóstico presuntivo según el sexo y la Gestión Académica.

\begin{tabular}{lccrrrrrrrrr}
\hline & \multicolumn{2}{c}{ Clase I } & \multicolumn{2}{c}{ Clase II-1 } & \multicolumn{2}{c}{ Clase II-2 } & \multicolumn{2}{c}{ Clase III } & \multicolumn{2}{c}{ N.A } \\
& $\mathrm{n}$ & $\%$ & $\mathrm{n}$ & \multicolumn{1}{c}{$\%$} & $\mathrm{n}$ & $\%$ & $\mathrm{n}$ & $\%$ & $\mathrm{n}$ & \multicolumn{1}{c}{$\%$} \\
\hline Femenino & 41 & 37 & 8 & 7 & 3 & 3 & 10 & 9 & 62 & 56 \\
Masculino & 32 & 29 & 8 & 7 & 3 & 3 & 10 & 5 & 49 & 44 \\
Total & 73 & 66 & 16 & 14 & 6 & 6 & 16 & 14 & 111 & 100 \\
\hline
\end{tabular}

Respecto a la clase esqueletal mediante el diagnóstico presuntivo, se registró más frecuente la clase I del sexo femenino con un $37 \%$ y la clase I del sexo masculino con un $29 \%$; en menor porcentaje se registró la clase II-2 con un $3 \%$ tanto en el sexo femenino como en el masculino. (Tabla 3)

\section{Discusión}

Los problemas de oclusión dental o maloclusiones son el resultado de la adaptación de la región orofacial a varios factores etiológicos que afectan la estética y funcionalidad del sistema estomatognático. ${ }^{4,5}$

Se han realizado varios estudios acerca de la prevalencia de maloclusiones en diversas poblaciones del mundo registrándose porcentajes que van desde un $39 \%$ hasta un $93 \%$, relacionando la variación a diferencias específicas en los grupos étnicos, número de sujetos incluidos en el estudio, edades y método de registro utilizado, siendo así su presencia en más del $60 \%$ de la población; registrándose la clase I como la de mayor frecuencia en relación a las clases II y III. ${ }^{3}$

En Latinoamérica se encuentra un alto índice de maloclusiones, en Perú, por ejemplo, Aliaga et al, ${ }^{4}$ registraron un $85.6 \%$, en una muestra de 201 escolares de los cuales 106 fueron mujeres; en cambio, Taboada et $\mathrm{al},{ }^{3}$ reconocieron una prevalencia catalogada como baja del $61 \%$, a pesar de haber realizado su estudio en comunidades nativas del oriente de México y con una muestra de 93 escolares de los cuales 49 fueron hombres y 44 mujeres.

El presente estudio realizado en 111 escolares de una parroquia urbana de Cuenca en donde 62 fueron mujeres y 49 varones ratificó esta tendencia, con un $77 \%$ indicando que es una enfermedad predominante. Un estudio realizado por Ourens et al, ${ }^{6}$ en el interior de Uruguay, con una muestra de 438 escolares de los cuales 234 fueron mujeres y 204 hombres registró una prevalencia del $33.8 \%$ ubicándose por debajo de los porcentajes establecidos a nivel mundial comprobándose que la presencia de maloclusiones se guía por la calidad de vida de los pacientes. ${ }^{3}$

Otros estudios se dividieron según la clasificaciónde Angle; Medina, ${ }^{14}$ con una muestra de 479 pacientes en su estudio realizado en Venezuela atribuye a la clase I con el $64.30 \%$ como la más prevalente en relación a las clase II que obtiene el $20.67 \%$ y por último registra a la clase III con el $15.03 \%$, además, su estudio reflejó que las maloclusiones son más frecuentes en hombres que en mujeres.

Plazas et al, ${ }^{17}$ en Cartajena de Indias, realizaron un estudio descriptivo con una muestra de 72 escolares de los cuales 34 fueron niños y 38 niñas obteniendo relaciones similares, pero ellos las clasificaron según el lado derecho e izquierdo, presentándose la clase I del lado derecho con el $58.83 \%$, y el lado izquierdo con el $62.50 \%$; la clase II del lado derecho con un $25 \%$ y el lado izquierdo con un $26.39 \%$ y en menor proporción la clase III del lado derecho con un $6.94 \%$ y el lado izquierdo con un $11.11 \%$.

Burgos, ${ }^{23}$ en un grupo étnico de Chile conformado por 184 estudiantes, registró a la Clase I como la más constante con un $51.1 \%$, seguida de la clase II con el $24.5 \%$; difiriendo con otros estudios en cuanto a la clase III ya que se registra bastante alta con un $21.7 \%$ atribuyéndose este porcentaje al grupo de estudio, además, no se encontró dimorfismo entre hombres y mujeres. En la India, se han registrado estudios con altos y bajos porcentajes de prevalencia de maloclusiones, Satinder et al, ${ }^{24}$ con una muestra de 691 pacientes, en la región de Leh, Jammu y Cachemira registraron a la clase I como la más frecuente con el $87.4 \%$, la clase II división I presentó el $8.7 \%$, luego se registró la clase III con un $2.5 \%$ y por último la clase II división II con el $1.4 \%$. Kumar et al, ${ }^{25}$ realizaron otro estudio en Pune, cinco escuelas del ejército fueron el grupo etareo, con 1200 escolares, 705 fueron hombres y 495 mujeres, encontrando a la Clase I más prevalente con el $75.2 \%$ en relación a la Clase II con el $23 \%$ y en menor proporción la Clase III con el $1.8 \%$ presentándose como en el estudio de Medina más frecuente en hombres que en mujeres.

Al igual que Burgos, en el Oeste de Nepal, Baral, ${ }^{26}$ no encontró un dimorfismo entre hombres y mujeres su estudio se realizó en 1284 personas de las cuales 656 fueron hombres y 628 mujeres, encontrándose la clase I como la más constante con el $71.5 \%$ seguida de la clase II división 1 con un $20.7 \%$, la clase III con un $4.1 \%$ y la clase II división 2 con el 3,9\%, registrándose en un orden similar 
al estudio realizado en Leh por Santinder y cols, y por el estudio realizado en la parroquia urbana Bellavista de la ciudad de Cuenca, donde se registró como más prevalente a la clase I con el $66 \%$, la clase II división 1 con un $14 \%$ igual que la clase III y en menor proporción se registró la clase II división 2 con un $6 \%$, ubicándose dentro del rango de investigaciones anteriores, pero difiriendo con ellas al relacionarlos según el género ya que se encontró mayor frecuencia en mujeres que en varones.

\section{Conclusión}

De 111 escolares examinados el $77 \%$ presentaron maloclusiones demostrando que es un enfermedad predominante en ésta parroquia. Al registrar las maloclusiones según el sexo se encontró mayor prevalencia en el sexo femenino con el $44 \%$ en relación a el sexo masculino que obtuvo el $37 \%$.

Según la relación molar de Angle en base al sexo, en el presente estudio se registró que el género femenino en la relación molar derecha, la clase I obtuvo un $41 \%$, la clase II $4 \%$, la clase III $7 \%$ y no se registró en el $4 \%$; en cambio en el sexo masculino la clase I registro el $29 \%$, la clase II $5 \%$, la clase III $5 \%$ y no se registró en el $5 \%$. En la relación molar del lado izquierdo el sexo femenino en la clase I obtuvo $37 \%$, la clase II $7 \%$, la clase III $9 \%$ y no se registró en el $3 \%$ en cambio en el sexo masculino la clase I obtuvo un $32 \%$, la clase II $5 \%$, la clase III $5 \%$ y no se registró en el $3 \%$, encontrando diferencias significativas en ambos sexos. De acuerdo a la prevalencia de la clase esqueletal mediante el diagnóstico presuntivo según el sexo, en el género femenino la clase I registró un $37 \%$, la clase II división I $7 \%$, la clase III $9 \%$ y la clase II división II $3 \%$ en cambio en el sexo masculino la clase I presentó un $29 \%$, la clase II división I $7 \%$, la clase III $5 \%$ y la clase II división 2 un $3 \%$, registrándose diferencias significativas en ambos sexos y mayor prevalencia de la clase I en relación a las clases II y III.

\section{Referencias Bibliográficas}

1. Okeson JP. Tratamiento de oclusión y afecciones temporomandibulares. London: Elsevier Health Sciences Spain; 2013. OCLC: 882778065. Available from: http://public. eblib.com/choice/publicfullrecord.aspx?p=1724173.

2. Leslie RRD, Erika ED, Antón-Sarabia J, Gabriel MQ. Asociación de maloclusiones clase I, II y III y su tratamiento en población infantil en la ciudad de Puebla, México. Revista Tamé. 2014;2(6):175-179.

3. Taboada Aranza O, Torres Zurita A, Cazares Martínez CE, Orozco Cuanalo L. Prevalencia de maloclusiones y trastornos del habla en una población preescolar del oriente de la Ciudad de México. Boletín médico del Hospital Infantil de México. 2011;68:425 - 430. Available from: http://www.scielo.org.mx/scielo.php?script= sci_arttext\&pid=S1665-11462011000600004\&nrm=iso.
4. Castillo AD, Mattos-Vela MA, Castillo-Mendoza D. Maloclusiones en niños y adolescentes de caseríos y comunidades nativas de la Amazonía de Ucayali, Perú. Revista Peruana de Medicina Experimental y Salud Pública. 2011;28(1):87-91.

5. Pincheira C, Thiers S, Bravo E, Olave H. Prevalencia de Maloclusiones en Escolares de 6 y 12 Años de Choshuenco - Neltume, Chile. International Journal of Medical and Surgical Sciences. 2016;3(2):829-837.

6. O M, C R, H JB, L S, N H, Á R, et al. Prevalencia de maloclusiones en adolescentes y adultos jóvenes del interior del Uruguay. Relevamiento nacional de salud bucal 2010-2011. ODONTOESTOMATOLOGÍA. 2013;XV:47-57. Available from: http://www.redalyc.org/ articulo.oa?id=479647723006.

7. Mata J, Zambrano O F adn Quirós, Maza PD, Jurisic A, Alced oC, Fuenmayor D, et al. Expansión rápida de Maxilar en Maloclusiones transversales: Revisión Bibliográfica. Revista Latinoamericana de Ortodoncia y Odontopediatría. 2009; Available from: https://www.ortodoncia. ws/publicaciones/2009/art-24/.

8. Palma Cárdenas A, Sánchez Aguilera F. Técnicas de ayuda odontológica y estomatológica. Madrid: Paraninfo; 2010. OCLC: 934357573.

9. Miriam Sada, Javier de Girón. Maloclusiones en la dentición temporal o mixta. Anales de Pediatría Continuada. 2006 Feb;4(1):66-70. Available from: http://linkinghub. elsevier.com/retrieve/pii/S1696281806735910.

10. Laura Cárabe Fernández. Análisis tridimensional de la inclinación bucolingual de primeros y segundos molares superiores en los distintos patrones faciales. [Trabajo de Fin de Máster en Ortodoncia y Ortopedia Dento-Facial]. Universidad de Oviedo; 2014. Available from: http://digibuo. uniovi.es/dspace/bitstream/10651/29600/6/Carabe.pdf.

11. Ebingen Villavicencio. Él tamaño muestral en tesis de postgrado. ¿cuántas personas debo encuestar? Unpublished; 2011. DOI: 10.13140/RG.2.1.3337.2243.

12. García García VJ V J, Ustrell Torrent JM, SentÃls Vilalta J. Evaluación de la maloclusión, alteraciones funcionales y hábitos orales en una población escolar: Tarragona y Barcelona. Avances en Odontoestomatología. 2011;27:75 - 84. Available from: http://scielo.isciii.es/scielo.php?script=sci_arttext\& pid=S0213-12852011000200003\&nrm=iso.

13. Mafla AC, Barrera DA, Mabel Muñoz G. Malocclusion and orthodontic treatment need in adolescents from Pasto, Colombia. Revista Facultad de Odontología Universidad de Antioquia. 2011;22(2):173-185. Available from: https://aprendeenlinea.udea.edu.co/revistas/ index.php/odont/article/view/7308/8880.

14. Medina AC. Prevalencia de maloclusiones dentales en un grupo de pacientes pediátricos. Acta Odontológica Venezolana. 2010;48:94-99.

5. Millán M, Katagiri M, Elorza H. Casuística de maloclusiones Clase I, Clase II y Clase III según Angle en el Departamento de Ortodoncia de la UNAM. Revista 
odontológica mexicana. 2007;11(4):175-180.

16. Morales FJU. Clasificación de la maloclusión en los planos anteroposterior, vertical y transversal. Revista ADM. 2007;64(3).

17. Plazas Román J, Martínez Bermúdez O, Castro Pacheco L, Solana García AM, Villalba Manotas LF. Prevalencia de maloclusiones en niños de una escuela en Cartagena de Indias. Ciencia y Salud Virtual. 2011;3(1):2. Available from: http://revistas.curn.edu.co/index.php/cienciaysalud/ article/view/38.

18. Mendoza Oropeza L. Prevalencia de las maloclusiones asociada con hábitos bucales nocivos en una muestra de mexicanos. Revista Mexicana de Ortodoncia. 2014;2(4ESP). Available from: http://revistas.unam.mx/index.php/ rmo/article/view/54211.

19. Vellini-Ferreira F. Ortodoncia: diagnóstico y planificación clínica. Sao Pablo: Artes Médicas; 2004. OCLC: 227035391.

20. Francisco MPJ, Alejandra CDP, Jasiel LA, José MDSM, Violeta ZM. Prevalencia de maloclusiones dentales en un grupo de adolescentes mexicanos y su relación con la edad y el género. Acta odontológica venezolana. 2007;45(1).

21. Uribe Restrepo GA. Ortodoncia: teoría y clínica. Medellín, Colombia: Corporación para Investigaciones Biológicas; 2010. OCLC: 781365799.

22. Botero Mariaca PM, Vélez Trujillo N. Manual de historia clínica odontológica del escolar. Universidad Cooperativa de Colombia; 2016. DOI: 10.16925/9789587600520. Available from: http:// ediciones.ucc.edu.co/index.php/ucc/catalog/book/70.

23. Burgos D. Prevalencia de Maloclusiones en Niños y Adolescentes de 6 a 15 Años en Frutillar, Chile. International journal of odontostomatology. 2014;8(1):13-19. Available from: http://www. scielo.cl/scielo.php?script=sci_arttext\&pid=S0718381X2014000100002\&lng=en\&nrm=iso\&tlng=en.

24. Singh SP, Kumar V, Narboo P. Prevalence of malocclusion among children and adolescents in various school of Leh Region. Journal of Orthodontics \& Endodontics. 2015;(2).

25. Kumar P, Londhe SM, Kotwal A, Mitra R. Prevalence of malocclusion and orthodontic treatment need in schoolchildren - An epidemiological study. Medical Journal Armed Forces India. 2013 Oct;69(4):369374. Available from: http://linkinghub.elsevier.com/ retrieve/pii/S0377123712000202.

26. Baral P. Prevalence of Malocclusion in Western Nepal. Orthodontic Journal of Nepal. 2016 Jul;5(2):6. Available from: http://www.nepjol.info/index.php/OJN/article/ view/15215.

Recibido: 30 de julio de 2017

Aceptado: 15 de agosto de 2017 
